\title{
Analysis of RF-DC Conversion Efficiency of Composite Multi-Antenna Rectifiers for Wireless Power Transfer
}

\author{
Chao Deng ${ }^{1}$, Kaibin Huang ${ }^{2}$, Yik-Chung $\mathrm{Wu}^{2}$, and Minghua Xia ${ }^{1}$ \\ ${ }^{1}$ School of Electronics and Information Technology, Sun Yat-sen University \\ Guangzhou, China \\ [E-mail : dengch9@mail2.sysu.edu.cn, xiamingh@mail.sysu.edu.cn] \\ ${ }^{2}$ Department of Electrical and Electronic Engineering, The University of Hong Kong \\ Hong Kong \\ [E-mail : \{huangkb, ycwu\}@eee.hku.hk] \\ *Corresponding author : Minghua Xia
}

Received May 1, 2017; revised August 29, 2017; accepted September 26, 2017; published October 31, 2017

\begin{abstract}
This paper studies the radio frequency to direct current (RF-DC) conversion efficiency of rectennas applicable to wireless power transfer systems, where multiple receive antennas are arranged in serial, parallel or cascaded form. To begin with, a $2.45 \mathrm{GHz}$ dual-diode rectifier is designed and its equivalent linear model is applied to analyze its output voltage and current. Then, using Advanced Design System (ADS), it is shown that the rectifying efficiency is as large as $66.2 \%$ in case the input power is $15.4 \mathrm{dBm}$. On the other hand, to boost the DC output, three composite rectennas are designed by inter-connecting two dual-diode rectifiers in serial, parallel and cascade forms; and their output voltage and current are investigated using their respective equivalent linear models. Simulation and experimental results demonstrate that all composite rectennas have almost the same RF-DC conversion efficiency as the dual-diode rectifier, yet the output of voltage or current can be significantly increased; in particular, the cascade rectenna obtains the highest rectifying efficiency.
\end{abstract}

Keywords: RF-DC conversion efficiency, series rectenna, parallel rectenna, cascade rectenna, wireless power transfer

A preliminary version of this paper was presented at ICONI 2016, and was selected as an outstanding paper. This work was supported in part by the National Natural Science Foundation of China (NSFC) under Grant No. 61671488 and in part by Special Fund for Science and Technology Development in Guangdong Province under Grants No. 2016A050503025 and No. 2016B010126003. 


\section{Introduction}

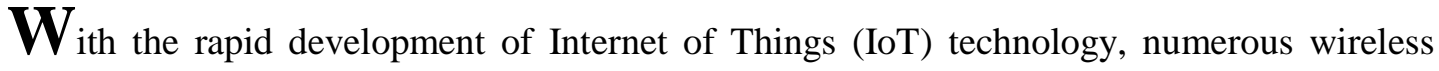
terminals with low-power consumptions are widely deployed in practice, e.g., sensors and radio frequency identification (RFID) tags. To resolve the painstaking power charging problem inherent in wireless terminals and the shortage of battery endurance, wireless power transfer (WPT) technique has received extensive attention from both academia and industry communities. To realize WPT in reality, many techniques like inductive coupling, resonant coupling, laser beaming and RF-based WPT have emerged in recent years. However, neither near-field coupling nor laser beaming is suitable for long-distance wireless power transfer, because of limited propagation distance and potential human safety concerns [1]. To speed up the application of RF-based WPT, improving the efficiency of RF power transfer is imperative.

The efficiency of a RF-based WPT system depends upon three parts: transmit (Tx) efficiency at the transmitter, free space propagation efficiency and receive (Rx) efficiency at the receiver [2]. In general, a rectenna consists of an Rx antenna and a rectifier and thus the Rx efficiency can be further decomposed into the antenna's Rx efficiency and rectifier's efficiency. To improve the antenna's Rx efficiency, there have been many publications focused on antenna quantity, polarization characteristic and/or array layout. For instance, in [3] a folded dipole antenna and a $3 \times 3$ array was designed and the effect of the number of antenna elements on Rx efficiency was explored. In [4], the performance of dual-patch, six-patch, and 16-patch antennas was extensively compared. In [5], a right-hand circularly polarized (RHCP) antenna and a left-hand circularly polarized (LHCP) antenna were designed, and the effect of polarization on antenna's Rx efficiency was investigated. For array layout, the effect of flat and random dispositions of rectifying antenna on the Rx efficiency was studied in [6]. Recently, interference inherent in wireless applications was seen as a new energy source which can be harvested by rectenna to improve the Rx efficiency [7][8].

To analyze the performance of rectifiers, several analytical models were proposed in the open literature, mainly including linear and nonlinear models. As for the linear one, there are two different approaches: one is to establish the relationship between input and output powers by use of the turn-on angle [9][10]; the other is to analyze the current by using the small signal model of diode [11]. Clearly, both approaches need to determine the optimal load prior to simulation experiments. On the other hand, for the nonlinear model, Ritz-Galerkin technique was widely used to investigate the closed-form of output voltage and it outperforms linear one in terms of accuracy at the cost of complexity [12][13]. In practice, if the load is already known, the linear rather than nonlinear mode is preferred.

As for the analytical linear models of composite rectennas, in [14] several kinds of rectenna arrays were analyzed, such as $\mathrm{H}-\mathrm{H}$ series, $\mathrm{H}-\mathrm{H}$ parallel, $\mathrm{H}-\mathrm{L}$ series, $\mathrm{H}-\mathrm{L}$ parallel, L-L series and L-L parallel, where " $\mathrm{H}$ " denotes the rectenna with higher output while " $\mathrm{L}$ " means the rectenna with lower output. The authors of [15] computed the resistance and inductance of transmission wire between the first stage and the second stage in cascade rectenna so as to explore the difference between cascade and series or parallel connection.

To perform theoretical analysis with equivalent circuit, simple linear models are developed in this paper to describe the output characteristics of two-stage series/parallel/cascade rectennas. We start with a basic dual-diode rectifier and then three composite rectennas are developed, by arranging two dual-diode rectifiers in serial, parallel and cascaded forms. Our analytical and experimental results show that the rectifying efficiency of the dual-diode 
rectifier can reach as high as $73.9 \%$, and the composite arrays enables boost the output current or voltage without degrading the rectifying efficiency. In particular, series rectenna gets its maximum conversion efficiency as high as $76.7 \%$, and exports twice of voltage than the single rectenna while they have almost the same output current. The maximum conversion efficiency of parallel rectenna is $78 \%$, and it has double output current than the single rectenna while they have almost the same output voltage. Among the four rectennas under study, the cascade rectenna obtains the highest conversion efficiency being $79.2 \%$.

The rest of this paper is organized as follows. In Section 2, after introducing the small signal model of diode, the performance of dual-diode rectifying circuit is analytically investigated. Then, Section 3 analyzes the rectifying efficiency of three composite multi-antenna rectifying circuits, including series, parallel and cascade rectennas. Both simulation results in ADS and physical experimental results collaborate the analysis. Finally, Section 4 concludes the paper.

\section{RF-DC Conversion Efficiency of Dual-Diode Rectifying Circuit}

Since dual-diode rectifier is a basic composition unit of composite multi-antenna rectennas to be investigated later, this section is dedicated to analyzing the rectifying efficiency of dual-diode rectifier, based on the small signal model of diode.

\subsection{The Small Signal Model of Diode}

As illustrated in Fig. 1, the small signal model of diode consists of four parts: an ideal diode $\mathrm{D}_{\mathrm{i}}$, a serial resistance $R_{s}$, a parallel capacitance $C_{j}$, and a parallel resistance $R_{j}$. Compared with $R_{j}$, the voltage drop on $R_{s}$ is generally very small and can be ignored in practice [11]. To establish the relationship between the input and output voltages, the currents through $D_{i}, C_{j}$ and $R_{j}$ are explicitly computed as follows.

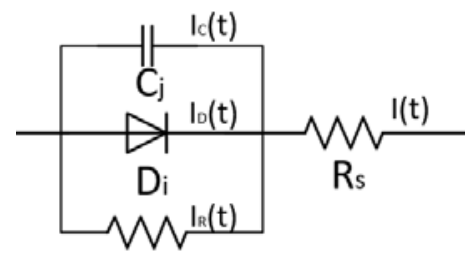

$\mathrm{R}_{\mathrm{j}}$

Fig. 1. The small signal model of diode. by

To begin with, recalling that the volt-current (V-I) characteristic of an ideal diode is given

$$
I_{D}(t)=I_{S}\left(\exp \left(\frac{V_{D}(t)}{N V_{T}}\right)-1\right),
$$

where $I_{D}(t)$ denotes the current through $\mathrm{D}_{\mathrm{i}}, I_{S}$ means the saturation current, $V_{D}(t)$ stands for the applied voltage, $N$ is the ideal factor of the diode and $V_{T}$ represents the thermal voltage [16], the value of $C_{j}$ can be determined by

$$
C_{j}(t)=C_{j 0}\left(1-\frac{V_{D}(t)}{V_{J}}\right)^{-M},
$$


where $C_{j 0}$ stands for the zero-bias barrier capacitance, $V_{J}$ is the built-in voltage, $M$ is the capacitance gradient factor with a default value being 0.5. By expressing the barrier capacitance with respect to its harmonic components, we have

$$
C_{j}(t)=C_{j 0}+C_{1} \cos (\omega t-\phi)+C_{2} \cos (2 \omega t-\phi)+\cdots,
$$

where $\phi$ denotes the shift of incident power phase. In practice, to suppress the harmonic ones, a microstrip transmission line with a length $L \mathrm{~mm}$ is generally strung at the end of the diode. As a result, (3) reduces to

$$
C_{j}(t)=C_{j 0}+C_{1} \cos (\omega t-\phi) .
$$

On the other hand, it is straightforward that the current through barrier capacitance can be expressed as

$$
I_{C}(t)=\frac{\mathrm{d}\left(C_{j}(t) V_{D}(t)\right)}{\mathrm{d} t} .
$$

Afterwards, the current through $\mathrm{R}_{\mathrm{j}}$ is readily given by

$$
I_{R}(t)=\frac{V_{D}(t)}{R_{j}} .
$$

Finally, the current over $\mathrm{R}_{\mathrm{s}}$ is given by

$$
I(t)=I_{D}(t)+I_{C}(t)+I_{R}(t) .
$$

\subsection{The Model of Dual-Diode Rectifier}

Fig. 2 shows the circuit model of dual-diode rectifier. When the circuit reaches steady state, the voltage drop on the storage capacitor $C_{1}$ is known as equilibrium voltage, which is to be analyzed in the following.

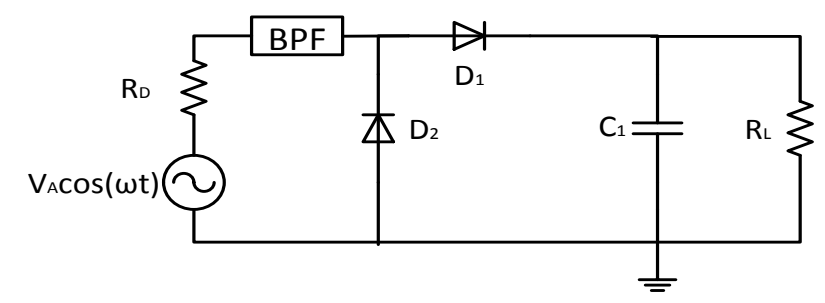

Fig. 2. Circuit model of dual-diode rectifier.

As shown in Fig. 2, the incoming RF voltage source is usually expressed as a cosine signal. In its positive half-cycle, diode $\mathrm{D}_{1}$ turns on whereas $\mathrm{D}_{2}$ cuts off. Consequently, the charges that enter $\mathrm{C}_{1}$ can be computed by integration as

$$
\Delta Q_{i n}=\int_{0}^{\frac{T}{2}} I_{D}(t) d t+\int_{0}^{\frac{T}{2}} I_{C}(t) d t+\int_{0}^{\frac{T}{2}} I_{R}(t) d t,
$$

where $T$ denotes the cycle of RF signals and $I_{D}, I_{C}$ and $I_{R}$ are previously given by (1), (5) and (6), respectively. Since $\mathrm{R}_{\mathrm{j}}$ is usually as large as hundreds of megaohm, $I_{R}(t)$ can be approximated to zero and, thus, the third term on the right-hand side (RHS) of (7) is negligible. The first term on the RHS of (7) is 


$$
\begin{aligned}
& \int_{0}^{\frac{T}{2}} I_{D}(t) d t=\int_{0}^{\frac{T}{2}} I_{S}\left(\exp \left(\frac{V_{D}(t)}{N V_{T}}\right)-1\right) d t \\
& =I_{S} \exp \left(-\frac{V_{C}}{N V_{T}}\right) \int_{0}^{T / 2} \exp \left(\frac{V_{A} \cos (\omega t)}{N V_{T}}\right) d t+\frac{T I_{S}}{2} \\
& =\frac{I_{S} T}{2}\left(\exp \left(\frac{-V_{C}}{N V_{T}}\right) I_{0}\left(\frac{V_{A}}{N V_{T}}\right)-1\right)
\end{aligned}
$$

where $I_{0}(z)$ denotes the zero-order modified Bessel function of first kind [17]. Finally, the second term on the RHS of (7) is readily computed as

$$
\int_{0}^{\frac{T}{2}} I_{C}(t) d t=2 C_{j 1} V_{C}-2 V_{j 0} V_{A} .
$$

In the negative half-cycle of the source, diode $\mathrm{D}_{1}$ cuts off whereas $\mathrm{D}_{2}$ turns on. At this time, the impedance of $D_{1}$ is very large so as to reject current released from storage capacitor. Therefore, the entire current will flow towards the terminal, and the charges released from the capacitor in this half-cycle is computed as

$$
\Delta Q_{\text {out }}=\int_{\frac{T}{2}}^{T} \frac{V_{C}}{R_{L}} d t=\frac{V_{C} T}{2 R_{L}} .
$$

When the charges entering the capacitor equals those leaving it, it reaches steady state, and we use $\tilde{V}_{C}$ instead of $V_{C}$ to represent the output voltage in the steady state. In other words, we have

$$
\Delta Q_{\text {in }}=\Delta Q_{\text {out }},
$$

which implies

$$
\frac{I_{S} T}{2}\left(\exp \left(\frac{-\tilde{V}_{C}}{N V_{T}}\right) I_{0}\left(\frac{V_{A}}{N V_{T}}\right)-1\right)+2 C_{j 1} \tilde{V}_{C}-2 C_{j 0} V_{A}=\frac{\tilde{V}_{C} T}{2 R_{L}}
$$

After performing some algebraic manipulations, (12) can be simplified as

$$
\frac{\frac{I_{S} T}{2} \exp \left(\frac{-\tilde{V}_{C}}{N V_{T}}\right) I_{0}\left(\frac{V_{A}}{N V_{T}}\right)}{2 C_{j 0} V_{A}+\frac{I_{S} T}{2}}=\frac{\tilde{V}_{C}\left(\frac{T}{2 R_{L}}-2 C_{j 1}\right)}{2 C_{j 0} V_{A}+\frac{I_{S} T}{2}}+1 .
$$

Taking natural logarithm on both sides of (13) yields

$$
\ln \frac{I_{S} T}{2}+\ln I_{0}\left(\frac{V_{A}}{N V_{T}}\right)-\ln \left(2 C_{j 0} V_{A}+\frac{I_{S} T}{2}\right)+\frac{-\tilde{V}_{C}}{N V_{T}}=\ln \left(\frac{\tilde{V}_{C}\left(\frac{T}{2 R_{L}}-2 C_{j 1}\right)}{2 C_{j 0} V_{A}+\frac{I_{S} T}{2}}+1\right) .
$$


Next, taking proper values of parameters as specified in the next subsection, the first term involved in the logarithm function on the RHS of (14) can be approximately given by

$$
\tilde{V}_{C}\left(\frac{T}{2 R_{L}}-2 C_{j 1}\right) /\left(2 C_{j 0} V_{A}+\frac{I_{S} T}{2}\right) \approx 2 \times 10^{-3}<<1 .
$$

Finally, by using Taylor's series approximation to the RHS of (14), i.e., $\log (1+x) \approx x$, the equilibrium voltage can be finally obtained:

$$
\tilde{V}_{C}=\frac{2 C_{j 0} V_{A} N V_{T}}{\left(\frac{T}{2 R_{L}}-2 C_{j 1}\right) N V_{T}+2 C_{j 0} V_{A}+\frac{I_{S} T}{2}} \ln \left(\frac{\frac{I_{S} T}{2} \mathrm{I}_{0}\left(\frac{V_{A}}{N V_{T}}\right)}{2 C_{j 0} V_{A}+\frac{I_{S} T}{2}}\right) .
$$

Notice that (16) corresponds to the case that an ideal BPF is applied in Fig. 2. In practice, however, microstrip BPF is generally exploited, which may have the characteristics of capacitance due to its in-built physical structure. In such a case, (16) can be rewritten as

$$
\tilde{V}_{C}=\alpha \times \frac{2 C_{j 0} V_{A} N V_{T}}{\left(\frac{T}{2 R_{L}}-2 C_{j 1}\right) N V_{T}+2 C_{j 0} V_{A}+\frac{I_{S} T}{2}} \ln \left(\frac{\frac{I_{S} T}{2} \cdot I_{0}\left(\frac{V_{A}}{N V_{T}}\right)}{2 C_{j 0} V_{A}+\frac{I_{S} T}{2}}\right),
$$

where $\alpha>1$ denotes the voltage amplification factor. When the capacitance of microstrip BPF equals $C_{1}$, the circuit shown in Fig. 2 is equivalent to a double-voltage rectifier with $\alpha=2$.

\subsection{Simulation Results}

To investigate the output performance of dual-diode rectifier, simulation tests are performed in ADS.

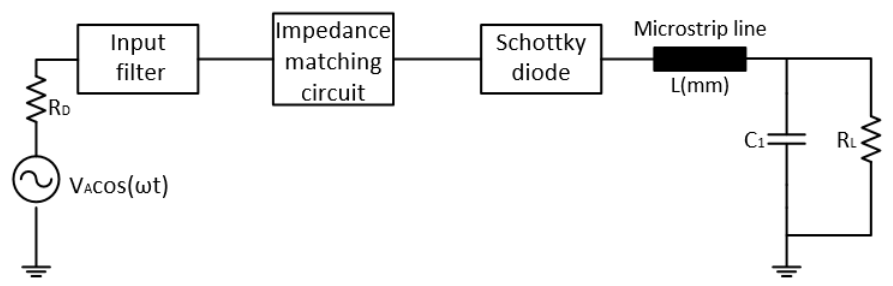

Fig. 3. Rectenna structure 


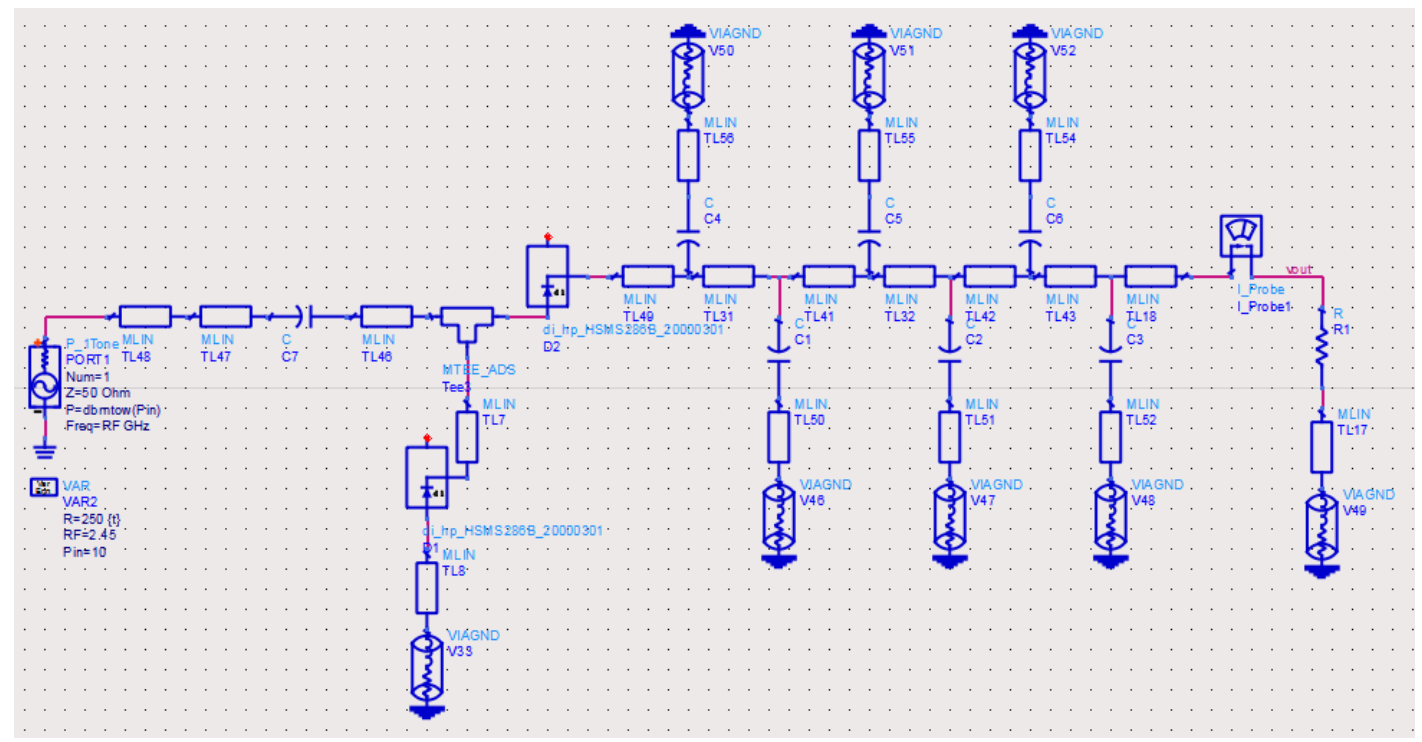

Fig. 4. The layout of a basic dual-diode rectifier in ADS.

Fig. 3 shows the structure of a basic single-element rectenna used in our simulation tests, which consists of eight parts: an RF source $V_{A} \cos (\omega t)$, an equivalent resistance of antenna $\mathrm{R}_{\mathrm{D}}$, an input filter, an impedance matching circuit, a Schottky diode (which is implemented by double diodes), an microstrip transmission line, a storage capacitor $\mathrm{C}_{1}$ and a load resistance $\mathrm{R}_{\mathrm{L}}$ [18]. With proper choice of frequency $2.45 \mathrm{GHz}$, dielectric substrate (F4B), Schottky diode (HSMS-286B), storage capacity (100 pF), and load resistance (250 Ohm), the PCB layout of the circuit is shown in Fig. 4. After performing some design optimization, our simulation results show that the maximum RF-DC conversion efficiency is $66.2 \%$ in the case of the input power being $15.4 \mathrm{dBm}$ (cf. Fig. 9). Correspondingly, the DC output voltage and current are $2.395 \mathrm{~V}$ and $10 \mathrm{~mA}$, respectively, (cf. Figs. 10-11).

For comparison purposes, Table 1 records the numerical results computed as per (16) compared with simulation results. In the simulation tests, the parameter setting includes $C_{j 0}=0.18 \mathrm{pF}, C_{j 1}=0.01 \mathrm{pF}, N=1.08, V_{T}=26 \mathrm{mV}, I_{S}=24.5 \mathrm{nA}$ and $T=1 /(2.45 \mathrm{GHz})$. It is observed that, when the input power is smaller than $10 \mathrm{dBm}$, the numerical results agree very well with simulation results. When the input power takes larger values, the difference between them becomes larger, due to the voltage amplification factor whose value change with different physical circuits and varied input powers.

Table 1. Comparison of numerical and simulation results.

\begin{tabular}{|c|c|c|c|}
\hline Pin (dBm) & $\mathbf{V}_{\mathbf{A}} \mathbf{( V )}$ & Numerical Results (V) & Simulation Results (V) \\
\hline 7 & 0.737 & 0.7009 & 0.766 \\
\hline 8 & 0.831 & 0.8726 & 0.836 \\
\hline 9 & 0.935 & 1.0651 & 1.020 \\
\hline 10 & 1.051 & 1.2822 & 1.171 \\
\hline 11 & 1.179 & 1.5240 & 1.342 \\
\hline 12 & 1.322 & 1.7962 & 1.534 \\
\hline
\end{tabular}




\section{RF-DC Conversion Efficiency of Composite Rectifying Circuits}

To improve the DC output, in this section three composite rectenna arrays are formed by series, parallel and cascade combinations, respectively. Simulation and experimental results show that the outputs of composite rectennas are significantly promoted, compared with the single dual-diode rectenna previously discussed.

For ease of subsequent comparison, the output current and voltage of the basic dual-diode rectifier shown in Fig. 2 are firstly given. Specifically, if the load is $R_{L}$, the output current and voltage, are easily expressed as

$$
\begin{gathered}
I=\frac{V_{0}}{R+R_{L}}, \\
V=\frac{V_{0} R_{L}}{R+R_{L}},
\end{gathered}
$$

where $V_{0}$ and $R$ are the equivalent voltage and resistance of rectifying circuit, respectively. When the rectifier achieves its maximum RF-DC conversion efficiency, $R$ must be equal to $R_{L}$. Next, the output current and voltage of three composite rectennas are examined, starting with the series combination.

\subsection{Series Combination}

Figs. 5-6 illustrate the model of series dual-diode rectenna combination and its equivalent linear circuit, respectively.

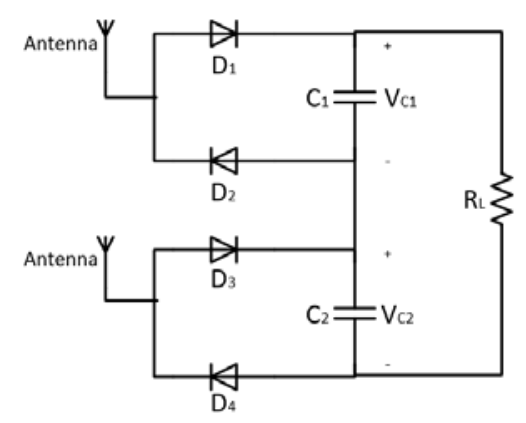

Fig. 5. The series combination of two rectennas.

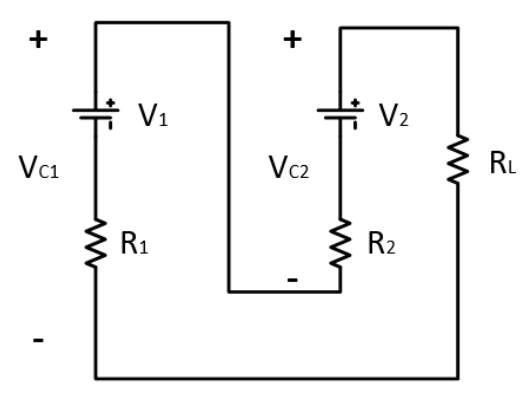

Fig. 6. The equivalent linear circuit of two serially combined rectennas [4]

Throughout this paper, it is assumed that two component dual-diode rectennas used to construct a composite rectenna (c.f. Fig. 5) are identical. With such an assumption, it is clear that $V_{1}=V_{2}$ and $R_{1}=R_{2}$. To maximize the output current, we must set $R_{L}=R_{1}+R_{2}$ and, thus, (20) - (21) can be rewritten as

$$
\begin{gathered}
I_{S}=\frac{1}{2}\left(I_{1}+I_{2}\right)=I_{1}, \\
V_{S}=V_{C 1}+V_{C 2}=2 V_{C} .
\end{gathered}
$$




\subsection{Parallel Combination}

Figs. 7-8 illustrate the model of parallel dual-diode recetenna combination and its equivalent linear circuit, respectively. By using a similar approach as above, the output current and voltage dropped on the load can be expressed as

$$
\begin{aligned}
& I_{P}=\frac{V_{1}+V_{2}}{2 R_{L}}=I_{1}+I_{2}=2 I_{1}, \\
& V_{P}=\frac{1}{2}\left(V_{C 1}+V_{C 2}\right)=V_{C},
\end{aligned}
$$

respectively, where $R_{L}=R_{1}=R_{2}$ was set to attain the maximum RF-DC conversion efficiency.

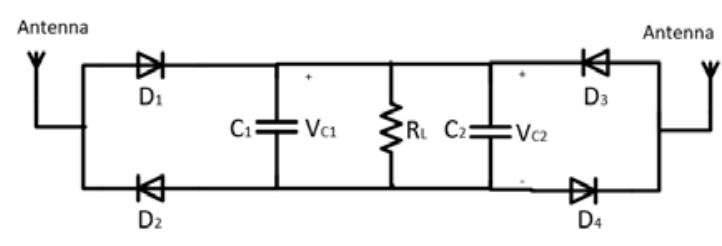

Fig. 7. The parallel combination of two dual-diode rectennas.

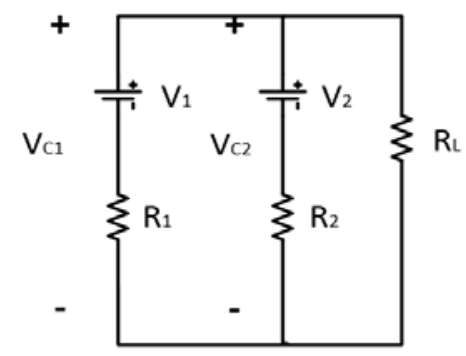

Fig. 8. The equivalent linear circuit of two rectennas combined in parallel.

\subsection{Cascade Combination}

Figs. 9-10 illustrate the model of cascade dual-diode recetenna combination and its equivalent linear circuit, respectively. Comparing Fig. 9 with Figs. 5 and 7 finds that the major difference between the cascade and series or parallel combination lies in that the output of the first stage serves as the source of the next stage. In this regards, the lower band pass filter (BPF) explicitly shown in Fig. 9 benefits preventing DC rectified by the upper stage flowing through the lower antenna. On the other hand, the low pass filter (LPF) in Fig. 9 is to ensure one-way transmission of the rectified energy. This LPF prevents the RF energy harvested by the lower antenna leaking to the upper stage without affecting DC produced by the upper stage. In practice, a non-ideal LPF with proper cutoff frequency benefits improving the rectifying efficiency by passing through a few residual harmonic components from the first stage to second stage. Those harmonic ones could be rectified again, thereby improving the DC output.

In theory, the cascade rectenna can be viewed as a composite series/parallel circuit [4]. In light of Fig. 10, the output current and voltage dropped on the load can be expressed as

$$
\begin{gathered}
I_{C}=I_{d c 2}+I_{h c}, \\
V_{C}=V_{C 2}=I_{C} \times R_{L},
\end{gathered}
$$


where $I_{d c 2}$ denotes the DC produced by the second stage and $I_{h c}$ refers to the harmonic components flowing towards the second stage due to the non-ideal characteristic of the LPF. In particular, it is straightforward that $I_{d c 1}=I_{d c 2}$ in ideal cases, namely, two identical rectennas are employed (cf. Fig. 9).

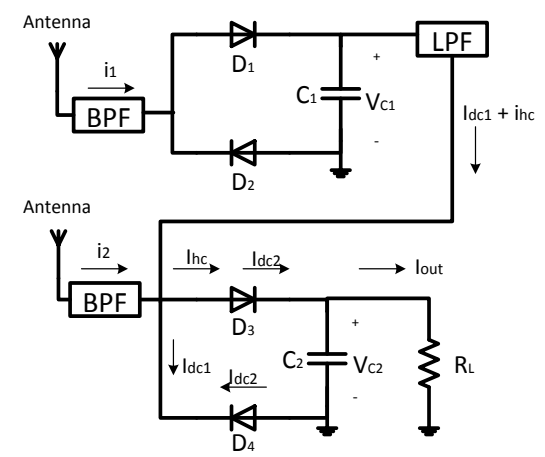

Fig. 9. The cascade combination of two dual-diode rectennas.

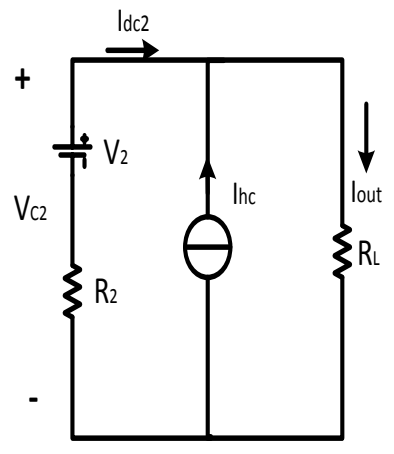

Fig. 10. The equivalent linear circuit of two rectennas combined in cascade.

It is remarkable that, in Fig. 9, the DC produced by the first-stage has no contribution to the final output since it will flow towards the ground via $\mathrm{D}_{4}$. This observation is verified in subsequent physical experiments by using a current detection probe.

\subsection{Simulation Results}

To verify the relationship shown in (22)-(27), three composite rectennas mentioned above are extensively simulated in ADS. In simulation setting, the parameters pertaining to RF source, dielectric substrate, Schottky diode, and storage capacity are identical to those listed in Section 2.3. The optimal loads of series, parallel and cascade rectenna are $400 \Omega, 150 \Omega$ and $250 \Omega$, respectively.

Figs. 11-13 shows the rectifying efficiency, output voltage and current versus the input power $P_{\text {in }}$ in the unit of $\mathrm{dBm}$, respectively. It is observed from Fig. 11 that, for the series combination, the maximum RF-DC conversion efficiency is $57 \%$ in the case of the input power being $15.8 \mathrm{dBm}$. Meanwhile, Figs. 12-13 show that the output voltage and current are $4.163 \mathrm{~V}$ and $10 \mathrm{~mA}$, respectively.

On the other hand, for the parallel combination, it is observed from Fig. 11 that the maximum conversion efficiency is $61.6 \%$ given that the input power is $16.1 \mathrm{dBm}$. Accordingly, the optimal output voltage and current are $2.24 \mathrm{~V}$ and $22 \mathrm{~mA}$, respectively (cf. Figs. 12-13).

As for the cascade rectenna, Fig. 11 shows that its rectifying efficiency performs the same as the other two in the low input power region, and the maximum conversion efficiency reaches $62.3 \%$ in the case of the input power being $15.4 \mathrm{dBm}$. Meanwhile, the DC output voltage and current are $3.287 \mathrm{~V}$ and $13 \mathrm{~mA}$, respectively (cf. Figs. 12-13). 


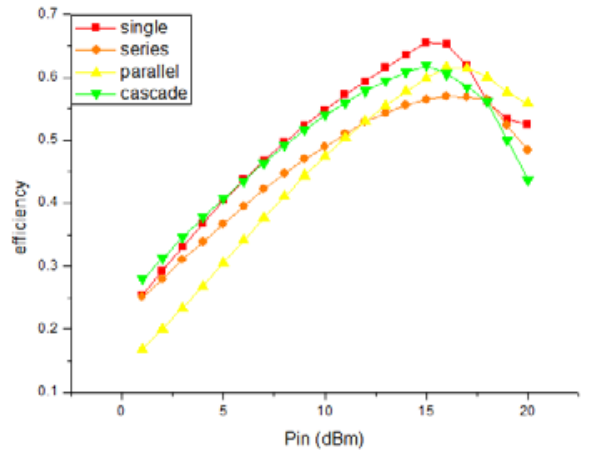

Fig. 11. Rectifying efficiency by simulation.

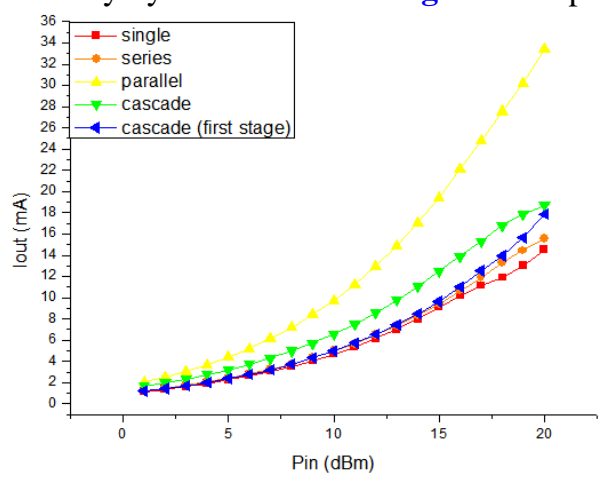

Fig. 13. Output current by simulation.

The blue curves shown in Fig. 12 and Fig. 13 represent the output voltage and current produced by the first-stage in a two-stage cascade rectenna. When the rectifying efficiency reaches the maximum, i.e., the input power is about $15.4 \mathrm{dBm}$, our simulation results show that the DC output current produced by the first-stage is $10 \mathrm{~mA}$ while the output at the second stage is $13 \mathrm{~mA}$, this additional $3 \mathrm{~mA}$ is due to harmonic components at the first stage, which corroborates the preceding analysis in Section 3.3.

\subsection{Experimental Results}

Apart from the preceding simulation results in ADS, to further verify the performance analysis of the developed rectennas, their PCBs are produced (as shown in Figs. 14-17) and extensive experimental tests are carried out.

Figs. 18-20 show the experimental results pertaining to the rectifying efficiency, DC output voltage and current versus the input power $P_{\text {in }}$ in the unit of $\mathrm{dBm}$, which are coincident with the simulation results shown in Figs. 11-13, respectively. In particular, the maximum rectifying efficiencies obtained in experimental tests are a bit larger than their respective simulation results. For instance, for the basic dual-diode rectenna, the maximum experimental rectifying efficiency is $73.92 \%$ in the case of the input power being $18 \mathrm{dBm}$ (cf. Fig. 19) whereas it is $56.1 \%$ by simulation (cf. Fig. 11). This is because the real-world PCB is optimized using a thin cover of copper foil. At the same time, the experimental output voltage and current of the basic dual-diode rectenna are $2.221 \mathrm{~V}$ and $21 \mathrm{~mA}$, respectively (cf. Figs. 19-20). 


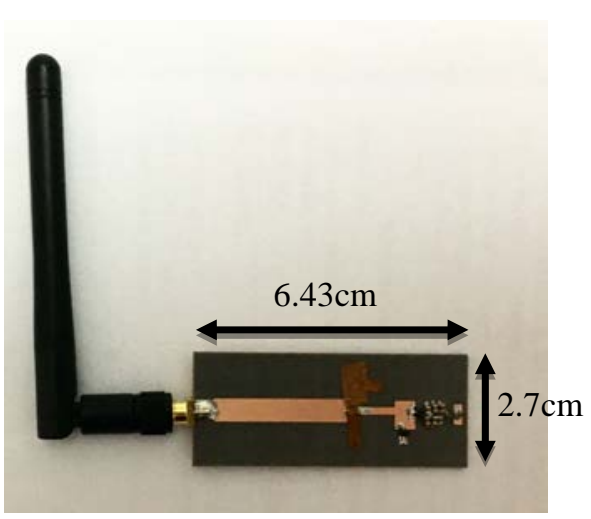

Fig. 14. PCB of single rectenna.

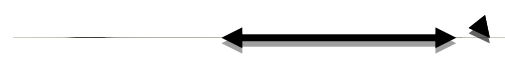

Fig. 15. PCB of serial rectenna.

$\Delta$

Fig. 16. PCB of parallel rectenna.

Fig. 17. PCB of cascade rectenna.

For the series rectenna, Fig. 18 shows that its maximum rectifying efficiency is $76.7 \%$ in the case of the input power being $18 \mathrm{dBm}$. At the same time, the output voltage and current are 4.27 V and $23 \mathrm{~mA}$, respectively (cf. Figs. 19-20). Clearly, this DC output voltage is almost twice of the basic dual-diode rectenna.

For the parallel rectenna, the maximum rectifying efficiency is $78 \%$ given that the input power is $19 \mathrm{dBm}$. Accordingly, the optimal DC output voltage and current are $2.58 \mathrm{~V}$ and 48 $\mathrm{mA}$, respectively (cf. Figs. 19-20). Clearly, its DC output current is almost twice of the single one.

As far as the cascade rectenna is concerned, its maximum rectifying efficiency is $79.2 \%$ in the case of the input power being $17.2 \mathrm{dBm}$ (cf. Fig. 18), which is $3.2 \%$ higher than that reported in [4]. Meanwhile, the DC output voltage and current are $2.771 \mathrm{~V}$ and $30 \mathrm{~mA}$, respectively (cf. Figs. 19-20). Therefore, it is obvious that the cascade rectenna outperforms the others in terms of the maximum rectifying efficiency. 


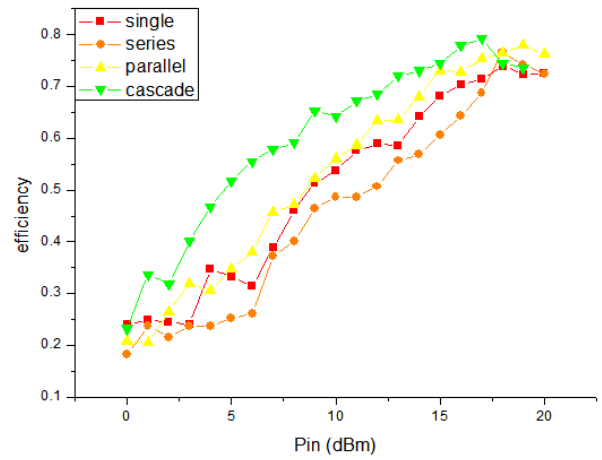

Fig. 18. Rectifying efficiency by experiment.

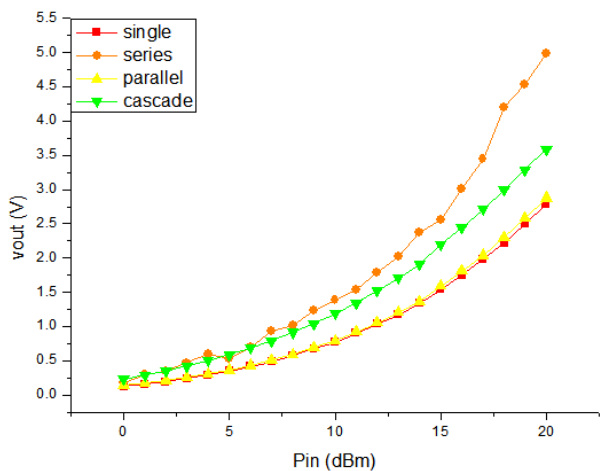

Fig. 19. DC output voltage by experiment.

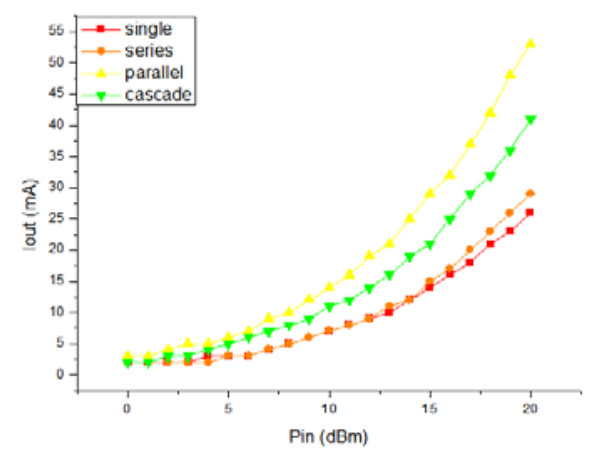

Fig. 20. DC output current by experiment.

\section{Conclusion}

In this paper, the rectifying efficiency of composite multi-antenna rectennas applicable to wireless power transfer was analytically studied. To start with, the output voltage and current of a basic dual-diode rectifier was firstly analyzed using the small signal model of diode. Then, a dual-diode rectenna operating at $2.45 \mathrm{GHz}$ was developed. To further boost the output current and/or voltage without degrading the rectifying efficiency, three composite rectennas were developed, by using series, parallel and cascaded combinations of two dual-diode rectifiers, our simulation and experimental results show that the series rectenna has the highest output voltage, the parallel rectenna gets the highest output current, while the cascade rectenna obtains the highest rectifying efficiency. These rectennas find extensive applications in wireless power transfer and/or energy harvesting terminals of low-power consumption, such as sensors and RFID tags.

\section{References}

[1] A. Massa, G. Oliveri, F. Viani, and P. Rocca, “Array designs for long- distance wireless power transmission: State-of-the-art and innovative solutions,” Proceedings of the IEEE, vol. 101, no. 6, pp. 1464-1481, 2013. Article (CrossRef Link)

[2] M. Xia and S. Aïssa, “On the efficiency of far-field wireless power transfer," IEEE Transactions on Signal Processing, vol. 63, no. 11, pp. 2835- 2847, 2015. Article (CrossRef Link)

[3] B. Strassner and K. Chang, "Highly efficiency-band circularly polarized rectifying antenna array for wireless microwave power transmission," IEEE Transactions on Antennas and Propagation, vol. 
51, no. 6, pp. 1347-1356, 2003. Article (CrossRef Link)

[4] Y. J. Ren and K. Chang, "5.8-GHz circularly polarized dual-diode rectenna and rectenna array for microwave power transmission," IEEE Transactions on Microwave Theory and Techniques, vol. 54, no. 4, pp. 1495-1502, 2006. Article (CrossRef Link)

[5] B. Strassner and K. Chang, "5.8-GHz circularly polarized dual-rhombic- loop traveling-wave rectifying antenna for low power-density wireless power transmission applications," IEEE Transactions on Microwave Theory and Techniques, vol. 51, no. 5, pp. 1548-1553, 2003.

Article (CrossRef Link)

[6] G. Franceschetti, A. Massa, and P. Rocca, "Innovative antenna systems for efficient microwave power collection," International Microwave Workshop Series on Innovative Wireless Power Transmission: Technologies, pp. 275-278, 2011. Article (CrossRef Link)

[7] N. Zhao, F. R. Yu, and V. C. M. Leung, "Wireless energy harvesting in interference alignment networks," IEEE Commun. Mag., vol. 53, no. 6, pp. 72-78, Jun. 2015. Article (CrossRef Link)

[8] N. Zhao, F. R. Yu, and V. C. M. Leung, "'Opportunistic communications in interference alignment networks with wireless power transfer," IEEE Wireless Commun., vol. 22, no. 1, pp. 88-95, Feb. 2015. Article (CrossRef Link)

[9] T. W. Yoo and K. Chang, "Theoretical and experimental development of $10 \mathrm{GHz}$ and $35 \mathrm{GHz}$ rectennas," IEEE Transactions on Microwave Theory and Techniques, vol. 40, no. 6, pp. 1259-1266, 1992. Article (CrossRef Link)

[10] M. Foruhandeh and S. Aïssa, "Efficiency of dc combination of rectified waveforms in energy harvesting systems," in Proc. of 2015 IEEE International Conference on Ubiquitous Wireless Broadband (ICUWB), 2015. Article (CrossRef Link)

[11] Y. Wu, J. P. Linnartz, H. Gao, and M. K. Matters-Kammerer, "Modeling of RF energy scavenging for batteryless wireless sensors with low input power," in Proc. of 2013 IEEE 24th Annual International Symposium on Personal, Indoor, and Mobile Radio Communications (PIMRC), pp. 527-531, 2013. Article (CrossRef Link)

[12] T. Miura, N. Shinohara, and H. Matsumoto, "Experimental study of rectenna connection for microwave power transmission," Electronics and Communications in Japan, vol. 84, no. 2, p. 2736, 2001. Article (CrossRef Link)

[13] S. Ladan and K. Wu, "Nonlinear modeling and harmonic recycling of millimeter-wave rectifier circuit," IEEE Transactions on Microwave Theory and Techniques, vol. 63, no. 3, pp. 937-944, 2015. Article (CrossRef Link)

[14] N. Shinohara and H. Matsumoto, "Dependence of DC output of a rectenna array on the method of interconnection of it’s array elements," Electrical Engineering in Japan, vol. 125, no. 1, pp. 9-17, 1998. Article (CrossRef Link)

[15] J.-W. Park, Y.-S. Kim, Y.-J. Yoon, J. Shin and J. So, "Rectifier conversion efficiency increase in low power using cascade connection at X-band," in Proc. of 2013 Proceedings of the International Symposium on Antennas \& Propagation, 2013. Article (CrossRef Link)

[16] B. R. Strickland and N. F. Audeh, "Circuit modeling of a diode-loaded dipole antenna," in Proc. of IEEE Energy and Information Technologies in the Southeast (Southeastcon '89), vol. 1, pp. 36-41, 1989. Article (CrossRef Link)

[17] F. Olver, D. Lozier, R. Boisvert, and C. Clark, NIST Handbook of Mathematical Functions, Cambridge University Press, 2010. Article (CrossRef Link)

[18] C. Deng, K. Huang, Y.-C. Wu, and M. Xia, "Analysis of RF-DC conversion efficiency in multi-antenna energy-harvesting receiver," in Proc. of The 8th International Conference on Internet (ICONI) 2016, pp. 211-216, Hong Kong, Dec. 16-19, 2016. 

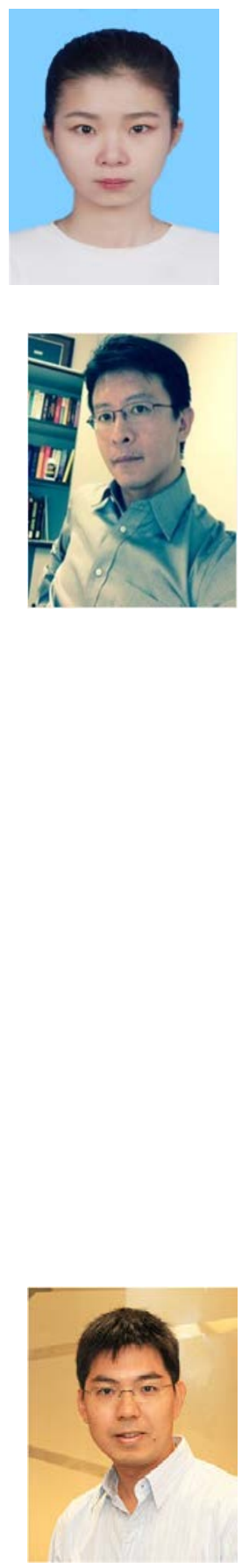

Kaibin Huang (M’08-SM'13) received the B.Eng. (Hons.) and M.Eng. degrees from the National University of Singapore, and the Ph.D. degree from The University of Texas at Austin (UT Austin), all in electrical engineering. He used to be a Faculty Member with the Department of Applied Mathematics (AMA), The Hong Kong Polytechnic University (PolyU), and the Department of EEE, Yonsei University. He has been a Post-Doctoral Research Fellow with the Department of Electrical and Computer Engineering, The Hong Kong University of Science and Technology, from 2008 to 2009, and an Associate Scientist with the Institute for Infocomm Research in Singapore, from 1999 to 2004. Since 2014, he has been an Assistant Professor with the Department of Electrical and Electronic Engineering (EEE), The University of Hong Kong. He is currently an Adjunct Professor with the School of EEE, Yonsei University, South Korea. His research interests focus on the analysis and design of wireless networks using stochastic geometry and multi-antenna techniques.

Dr. Huang is an Elected Member of the SPCOM Technical Committee of the IEEE Signal Processing Society. He serves on the technical program committees of major IEEE conferences in wireless communications. He has been the Technical Chair and Co-Chair for the IEEE CTW 2013, the Communication Theory Symposium of the IEEE GLOBECOM 2014, and the Advanced Topics in Wireless Communications Symposium of the IEEE /CIC ICCC 2014, and has been the Track Chair/Co-Chair for the IEEE PIMRC 2015, the IEE VTC Spring 2013, the Asilomar 2011, and the IEEE WCNC 2011. He is currently an Editor of the IEEE Journal on Selected Areas in Communications (JSAC) series on Green Communications and Networking, the IEEE Transactions on Wireless Communications, and the IEEE Wireless Communications Letters. He was also a Guest Editor for the JSAC special issues on communications powered by energy harvesting and an Editor of the IEEE/KICS JOURNAL OF COMMUNICATION AND NETWORKS (2009 through 2015). He received the 2015 IEEE ComSoc Asia Pacific Outstanding Paper Award, the Outstanding Teaching Award from Yonsei, Motorola Partnerships in Research Grant, the University Continuing Fellowship from UT Austin, and the Best Paper Award from the IEEE GLOBECOM 2006 and PolyU AMA in 2013.

Yik-Chung Wu received the B.Eng. (EEE) degree in 1998 and the M.Phil. degree in 2001 from the University of Hong Kong (HKU). He received the Croucher Foundation scholarship in 2002 to study Ph.D. degree at Texas A\&M University, College Station, and graduated in 2005. From August 2005 to August 2006, he was with the Thomson Corporate Research, Princeton, NJ, as a Member of Technical Staff. Since September 2006, he has been with HKU, currently as an Associate Professor. He was a visiting scholar at Princeton University, in summers of 2011 and 2015. His research interests are in general areas of signal processing, machine learning and communication systems, and in particular distributed signal processing, Bayesian inference, and robust optimization. Dr. Wu served as an Editor for IEEE Communications Letters, is currently an Editor for IEEE Transactions on Communications and Journal of Communications and Networks. 


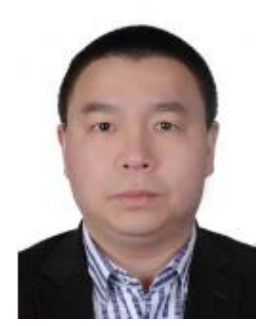

Minghua Xia (M’12) received the Ph.D. degree in telecommunications and information systems from Sun Yat-sen University, Guangzhou, China, in 2007, where since 2015, he has been working as a Professor.

From 2007 to 2009, he was with the Electronics and Telecommunications Research Institute (ETRI) of South Korea, Beijing R\&D Center, Beijing, China, where he worked as a member, and then, as a senior member of the engineering staff and participated in projects on the physical-layer design of 3GPP LTE mobile communications. From 2010 to 2014, he was in sequence with The University of Hong Kong, Hong Kong; King Abdullah University of Science and Technology, Jeddah, Saudi Arabia; and the Institut National de la Recherche Scientifique, University of Quebec, Montreal, QC, Canada, as a Postdoctoral Fellow. He holds two patents granted in China. His recent research interests focus on the design and analysis of wireless power transfer and/or energy harvesting systems.

Dr. Xia received the Professional Award at the 2015 IEEE TENCON in Macau, China. He also received an Exemplary Reviewer Award from the IEEE Transactions on Communications (2014), the IEEE Communications Letters (2014), and the IEEE Wireless CommSunications Letters $(2014,2015)$. 